\title{
Quantum Bohmian Model for Financial Market
}

\author{
Olga Al. Choustova \\ International Center for Mathematical Modeling \\ in Physics and Cognitive Sciences, \\ MSI, University of Växjö, S-35195, Sweden
}

\begin{abstract}
We apply methods of quantum mechanics for mathematical modeling of price dynamics at the financial market. The Hamiltonian formalism on the price/price-change phase space describes the classicallike evolution of prices. This classical dynamics of prices is determined by "hard" conditions (natural resources, industrial production, services and so on). These conditions are mathematically described by the classical financial potential $V(q)$, where $q=\left(q_{1}, \ldots, q_{n}\right)$ is the vector of prices of various shares. But the information exchange and market psychology play important (and sometimes determining) role in price dynamics. We propose to describe such behavioral financial factors by using the pilot wave (Bohmian) model of quantum mechanics. The theory of financial behavioral waves takes into account the market psychology. The real trajectories of prices are determined (through the financial analogue of the second Newton law) by two financial potentials: classical-like $V(q)$ ("hard" market conditions) and quantum-like $U(q)$ (behavioral market conditions).
\end{abstract}

Keywords: Quantum mechanics, financial market, information field of expectations, Bohmian mechanics, information pilot wave

\section{Introduction}

In economics and financial theory, analysts use random walk techniques to model behavior of asset prices, in particular share prices on stock markets, 
currency exchange rates and commodity prices. This practice has its basis in the presumption that investors act rationally and without bias, and that at any moment they estimate the value of an asset based on future expectations. Under these conditions, all existing information affects the price, which changes only when new information comes out. By definition, new information appears randomly and influences the asset price randomly. Corresponding continuous time models are based on stochastic processes (this approach was initiated in the thesis of L. Bachelier [1] in 1890), see, e.g., the book of R. N. Mantegna and H. E. Stanley 2 for historical and mathematical details.

However, empirical studies have demonstrated that prices do not completely follow random walk. Low serial correlations (around 0.05) exist in the short term; and slightly stronger correlations over the longer term. Their sign and the strength depend on a variety of factors, but transaction costs and bid-ask spreads generally make it impossible to earn excess returns. Interestingly, researchers have found that some of the biggest prices deviations from random walk result from seasonal and temporal patterns, see the book [2].

Therefore it would be natural to develop approaches which are not based on the assumption that investors act rationally and without bias and that, consequently, new information appears randomly and influences the asset price randomly. In particular, there are two well established (and closely related ) fields of research behavioral finance and behavioral economics which apply scientific research on human and social cognitive and emotional biases ${ }^{1}$ to better understand economic decisions and how they affect market prices, returns and the allocation of resources. The fields are primarily concerned with the rationality, or lack thereof, of economic agents. Behavioral models typically integrate insights from psychology with neo-classical economic theory. Behavioral analysis are mostly concerned with the effects of mar-

\footnotetext{
${ }^{1}$ Cognitive bias is any of a wide range of observer effects identified in cognitive science, including very basic statistical and memory errors that are common to all human beings and drastically skew the reliability of anecdotal and legal evidence. They also significantly affect the scientific method which is deliberately designed to minimize such bias from any one observer. They were first identified by Amos Tversky and Daniel Kahneman as a foundation of behavioral economics. Bias arises from various life, loyalty and local risk and attention concerns that are difficult to separate or codify. Tversky and Kahneman claim that they are at least partially the result of problem-solving using heuristics, including the availability heuristic and the representativeness.
} 
ket decisions, but also those of public choice, another source of economic decisions with some similar biases.

Since the 1970s, the intensive exchange of information in the world of finances has become one of the main sources determining dynamics of prices. Electronic trading (that became the most important part of the environment of the major stock exchanges) induces huge information flows between traders (including foreign exchange market). Financial contracts are performed at a new time scale that differs essentially from the old "hard" time scale that was determined by the development of the economic basis of the financial market. Prices at which traders are willing to buy (bid quotes) or sell (ask quotes) a financial asset are not more determined by the continuous development of industry, trade, services, situation at the market of natural resources and so on. Information (mental, market-psychological) factors play very important (and in some situations crucial) role in price dynamics. Traders performing financial operations work as a huge collective cognitive system. Roughly speaking classical-like dynamics of prices (determined) by "hard" economic factors is permanently perturbed by additional financial forces, mental (or market-psychological) forces, see the book of J. Soros [3].

In this paper we develop a new approach that is not based on the assumption that investors act rationally and without bias and that, consequently, new information appears randomly and influences the asset price randomly. Our approach can be considered as a special econophysical [2] model in the domain of behavioral finance. In our approach information about financial market (including expectations of agents of the financial market) is described by an information field $\psi(q)$ - financial wave. This field evolves deterministically ${ }^{2}$ perturbing the dynamics of prices of stocks and options. Since psychology of agents of the financial market gives an important contribution into the financial wave $\psi(q)$, our model can be considered as a special psycho-financial model.

This paper can be also considered as a contribution into applications of quantum mechanics outside microworld, see also books [4], [5]. The complete version of the present paper was submitted as the quant-preprint [6]. This paper is fundamentally based on investigations of D. Bohm, B. Hiley, and P. Pylkkänen [12, 13] on the active information interpretation of Bohmian mechanics [14, [15] and its applications to cognitive sciences, see also Khrennikov [16], [17].

\footnotetext{
${ }^{2}$ Dynamics is given by Schrödinger's equation on the space of prices of shares.
} 
There were performed numerous investigations on applying quantum methods to financial market, see, e.g., E. Haven [7- 9], that were not directly coupled to behavioral modeling, but based on the general concept that randomness of the financial market can be better described by the quantum mechanics, see, e.g., [10]: "A natural explanation for extreme irregularities in the evolution of prices in financial markets is provided by quantum effects."

\section{Financial phase-space}

Let us consider a mathematical model in that a huge number of agents of the financial market interact with one another and take into account external economic (as well as political, social and even meteorological) conditions in order to determine the price to buy or sell financial assets. We consider the trade with shares of some corporations (e.g., VOLVO, SAAB, IKEA,...). ${ }^{3}$

We consider a price system of coordinates. We enumerate corporations which did emissions of shares at the financial market under consideration: $j=1,2, \ldots, n$ (e.g., VOLVO: $j=1$, SAAB: $j=2$, IKEA: $j=3, \ldots$ ). There can be introduced the $n$-dimensional configuration space $Q=R^{n}$ of prices, $q=\left(q_{1}, \ldots, q_{n}\right)$, where $q_{j}$ is the price of a share of the $j$ th corporation. Here $R$ is the real line. Dynamics of prices is described by the trajectory $q(t)=\left(q_{1}(t), \ldots, q_{n}(t)\right)$ in the configuration price space $Q$.

Another variable under the consideration is the price change variable: $v_{j}(t)=\dot{q}_{j}(t)=\lim _{\Delta t \rightarrow 0} \frac{q_{j}(t t \Delta t)-q_{j}(t)}{\Delta t}$, see, for example, the book [2] on the role of the price change description. In real models we consider the discrete time scale $\Delta t, 2 \Delta t, \ldots$. Here we should use discrete price change variable $\delta q_{j}(t)=q_{j}(t+\Delta t)-q_{j}(t)$.

We denote the space of price changes by the symbol $V\left(\equiv R^{n}\right), v=$ $\left(v_{1}, \ldots, v_{n}\right)$. As in classical physics, it is useful to introduce the phase space $Q \times V=R^{2 n}$, namely the price phase space. A pair $(q, v)=$ (price, price change) is called a state of the financial market. (Later we shall consider quantum-like states of the financial market. A state $(q, v)$ is a classical state.)

We now introduce an analogue $m$ of mass as the number of items (in our case shares) that a trader emitted to the market. ${ }^{4}$ We call $m$ the financial

\footnotetext{
${ }^{3}$ Similar models can be developed for trade with options, see E. Haven [11 for the Bohmian financial wave model for portfolio.

${ }^{4}$ 'Number' is a natural number $m=0,1, \ldots,-$ the price of share, e.g., in the US-dollars. However, in a mathematical model it can be convenient to consider real $\mathrm{m}$. This can be
} 
mass. Thus each trader $j$ (e.g., VOLVO) has its own financial mass $m_{j}$ (the size of the emission of its shares). The total price of the emission performed by the $j$ th trader is equal to $T_{j}=m_{j} q_{j}$. Of course, it depends on time: $T_{j}(t)=m_{j} q_{j}(t)$. To simplify considerations, we consider a market at that any emission of shares is of the fixed size, so $m_{j}$ does not depend on time. In principle, our model can be generalized to describe a market with timedependent financial masses, $m_{j}=m_{j}(t)$.

We also introduce financial energy of the market as a function $H$ : $Q \times V \rightarrow R$. If we use the analogue with classical mechanics. (Why not? In principle, there is not so much difference between motions in "physical space" and "price space".), then we could consider (at least for mathematical modeling) the financial energy of the form:

$$
H(q, p)=\frac{1}{2} \sum_{j=1}^{n} m_{j} v_{j}^{2}+V\left(q_{1}, \ldots, q_{n}\right)
$$

Here $K=\frac{1}{2} \sum_{j=1}^{n} m_{j} v_{j}^{2}$ is the kinetic financial energy and $V\left(q_{1}, \ldots, q_{n}\right)$ is the potential financial energy, $m_{j}$ is the financial mass of $j$ th trader. ${ }^{5}$

The potential financial energy $V$ describes the interactions between traders $j=1, \ldots ., n$ (e.g., competition between NOKIA and ERICSSON) as well as external economic conditions (e.g., the price of oil and gas) and even meteorological conditions (e.g., the weather conditions in Louisiana and Florida). For example, we can consider the simplest interaction potential: $V\left(q_{1}, \ldots, q_{n}\right)=$ $\sum_{j=1}^{n}\left(q_{i}-q_{j}\right)^{2}$. The difference $\left|q_{1}-q_{j}\right|$ between prices is the most important condition for arbitrage.

We could never take into account all economic and other conditions that have influences to the market. Therefore by using some concrete potential $V(q)$ we consider the very idealized model of financial processes. However, such an approach is standard for physical modeling where we also consider idealized mathematical models of real physical processes.

useful for transitions from one currency to another.

${ }^{5}$ The kinetic financial energy represents efforts of agents of financial market to change prices: higher price changes induce higher kinetic financial energies. If the corporation $j_{1}$ has higher financial mass than the corporation $j_{2}$, so $m_{j_{1}}>m_{j_{2}}$, then the same change of price, i.e., the same financial velocity $v_{j_{1}}=v_{j_{2}}$, is characterized by higher kinetic financial energy: $K_{j_{1}}>K_{j_{2}}$. We also remark that high kinetic financial energy characterizes rapid changes of the financial situation at market. However, the kinetic financial energy does not give the attitude of these changes. It could be rapid economic growth as well as recession. 
We apply the Hamiltonian dynamics on the price phase space. As in classical mechanics for material objects, we introduce a new variable $p=$ $m v$, the price momentum variable. Instead of the price change vector $v=$ $\left(v_{1}, \ldots, v_{n}\right)$, we consider the price momentum vector $p=\left(p_{1}, \ldots, p_{n}\right), p_{j}=$ $m_{j} v_{j}$. The space of price momentums is denoted by the symbol $P$. The space $\Omega=Q \times P$ will be also called the price phase space. Hamiltonian equations of motion on the price phase space have the form: $\dot{q}=\frac{\partial H}{\partial p_{j}}, \dot{p}_{j}=-\frac{\partial H}{\partial q_{j}}, j=$ $1, \ldots, n$.

If the financial energy has form (11) then the Hamiltonian equations have the form $\dot{q}_{j}=\frac{p_{j}}{m_{j}}=v_{j}, \dot{p}_{j}=-\frac{\partial V}{\partial q_{j}}$. The latter equation can be written in the form: $m_{j} \dot{v}_{j}=-\frac{\partial V}{\partial q_{j}}$. The quantity $\dot{v}_{j}=\lim _{\Delta t \rightarrow 0} \frac{v_{j}(t+\Delta t)-v_{j}(t)}{\Delta t}$ is natural to call the price acceleration (change of price change). The quantity $f_{j}(q)=-\frac{\partial V}{\partial q_{j}}$ is called the (potential) financial force. We get the financial variant of the second Newton law:

$$
m \dot{v}=f
$$

"The product of the financial mass and the price acceleration is equal to the financial force."

In fact, the Hamiltonian evolution is determined by the following fundamental property of the financial energy: The financial energy is not changed in the process of Hamiltonian evolution: $H\left(q_{1}(t), \ldots, q_{n}(t), p_{1}(t), \ldots, p_{n}(t)=\right.$ $H\left(q_{1}(0), \ldots q_{n}(0), p_{1}(0), \ldots, p_{n}(0)\right)$. We need not restrict our considerations to financial energies of form (11). First of all external (e.g. economic) conditions as well as the character of interactions between traders at the market depend strongly on time. This must be taken into account by considering time dependent potentials: $V=V(t, q)$. Moreover, the assumption that the financial potential depends only on prices, $V=V(t, q)$, in not so natural for the modern financial market. Financial agents have the complete information on price changes. This information is taken into account by traders for acts of arbitrage, see [2] for the details. Therefore, it can be useful to consider potentials that depend not only on prices, but also on price changes: $V=V(t, q, v)$ or in the Hamiltonian framework: $V=V(t, q, p)$. In such a case the financial force is not potential. Therefore, it is also useful to consider the financial second Newton law for general financial forces: $m \dot{v}=f(t, q, p)$.

Remark 1. (On the form of the kinetic financial energy) We copied the form of kinetic energy from classical mechanics for material objects. It may be that such a form of kinetic financial energy is not justified by real financial market. It might be better to consider our choice of the kinetic 
financial energy as just the basis for mathematical modeling (and looking for other possibilities).

Remark 2. (Domain of price-dynamics) It is natural to consider a model in that all prices are nonnegative, $q_{j}(t) \geq 0$. Therefore financial Hamiltonian dynamics should be considered in the phase space $\Omega_{+}=R_{+}^{n} \times R^{n}$, where $R_{+}$is the set of nonnegative real numbers. We shall not study this problem in details, because our aim is the study of the corresponding quantum dynamics. But in the quantum case this problem is solved easily. One should just consider the corresponding Hamiltonian in the space of square integrable functions $L_{2}\left(\Omega_{+}\right)$. Another possibility in the classical case is to consider centered dynamics of prices: $z_{j}(t)=q_{j}(t)-q(0)$. The centered price $z_{j}(t)$ evolves in the configuration space $R^{n}$.

\section{Classical dynamics does not describe the real stock market}

The model of Hamiltonian price dynamics on the price phase space can be useful to describe a market that depends only on "hard" economic conditions: natural resources, volumes of production, human resources and so on. However, the classical price dynamics could not be applied (at least directly) to modern financial markets. It is clear that the stock market is not based only on these "hard" factors. There are other factors, soft ones (behavioral), that play the important and (sometimes even determining) role in forming of prices at the financial market. Market's psychology should be taken into account. Negligibly small amounts of information (due to the rapid exchange of information) imply large changes of prices at the financial market. We can consider a model in that financial (psychological) waves are permanently present at the market. Sometimes these waves produce uncontrollable changes of prices disturbing the whole market (financial crashes). Of course, financial waves also depend on "hard economic factors." However, these factors do not play the crucial role in forming of financial waves. Financial waves are merely waves of information. ${ }^{6}$

\footnotetext{
${ }^{6}$ We could compare behavior of financial market with behavior of a gigantic ship that is ruled by a radio signal. A radio signal with negligibly small physical energy can essentially change (due to information contained in this signal) the motion of the gigantic ship. If we do not pay attention on (do not know about the presence of) the radio signal, then we will be continuously disappointed by ship's behavior. It can change the direction of
} 


\section{Financial pilot waves}

If we interpret the pilot wave as a field, then we should pay attention that this is a rather strange field. It differs crucially from "ordinary physical fields," i.e., the electromagnetic field. We mention some o pathological features of the pilot wave field, see [14, 12, [15] for the detailed analysis. In particular, the force induced by this pilot wave field does not depend on the amplitude of wave. Thus small waves and large waves equally disturb the trajectory of an elementary particle. Such features of the pilot wave give the possibility to speculate, see [12, 13, that this is just a wave of information (active information). Hence, the pilot wave field describes the propagation of information. The pilot wave is more similar to a radio signal that guides a ship. Of course, this is just an analogy (because a radio signal is related to an ordinary physical field, namely, the electromagnetic field). The more precise analogy is to compare the pilot wave with information contained in the radio signal.

We remark that the pilot wave (Bohmian) interpretation of quantum mechanics is not the conventional one. As we have already noted, there are a few critical arguments against Bohmian quantum formalism:

1. Bohmian theory gives the possibility to provide the mathematical description of the trajectory $q(t)$ of an elementary particle. However, such a trajectory does not exist according to the conventional quantum formalism.

2. Bohmian theory is not local, namely, via the pilot wave field one particle "feels" another on large distances.

We say that these disadvantages of theory will become advantages in our applications of Bohmian theory to financial market. We also recall that already Bohm and Hiley [12] and Hiley and Pilkkänen 13 discussed the possibility to interpret the pilot wave field as a kind of information field. This information interpretation was essentially developed in works of Khrennikov [16], 17] that were devoted to pilot wave cognitive models.

motion without any "hard" reason (weather, destination, technical state of ship's equipment). However, if we know about the existence of radio monitoring, then we could find information that is sent by radio. This would give us the powerful tool to predict ship's trajectory. We now inform the reader that this example on ship's monitoring was taken from the book of D. Bohm and B. Hiley [12] on so called pilot wave quantum theory (or Bohmian quantum mechanics). 
Our fundamental assumption is that agents at the modern financial market are not just "classical-like agents." Their actions are ruled not only by classical-like financial potentials $V\left(t, q_{1}, \ldots, q_{n}\right)$, but also (in the same way as in the pilot wave theory for quantum systems) by an additional information (or psychological) potential induced by a financial pilot wave.

Therefore we could not use the classical financial dynamics (Hamiltonian formalism) on the financial phase space to describe the real price trajectories. Information (psychological) perturbation of Hamiltonian equations for price and price change must be taken into account. To describe such a model mathematically, it is convenient to use such an object as a financial pilot wave that rules the financial market.

In some sense $\psi(q)$ describes the psychological influence of the price configuration $q$ to behavior of agents of the financial market. In particular, the $\psi(q)$ contains expectations of agents. ${ }^{7}$

We underline two important features of the financial pilot wave model:

1. All shares are coupled on the information level. The general formalism [14], [12, [15] of the pilot wave theory says that if the function $\psi\left(q_{1}, \ldots, q_{n}\right)$ is not factorized, i.e.,

$$
\psi\left(q_{1}, \ldots, q_{n}\right) \neq \psi_{1}\left(q_{1}\right) \ldots \psi_{n}\left(q_{n}\right),
$$

then any changing the price $q_{i}$ will automatically change behavior of all agents of the financial market (even those who have no direct coupling with $i$-shares). This will imply changing of prices of $j$-shares for $i \neq j$. At the same time the "hard" economic potential $V\left(q_{1}, \ldots, q_{n}\right)$ need not contain any interaction term: for example, $V\left(q_{1}, \ldots, q_{n}\right)=q_{1}^{2}+\ldots q_{n}^{2}$. The Hamiltonian equations in the absence of the financial pilot wave have the form: $\dot{q}_{j}=$ $p_{j}, \dot{p}_{j}=-2 q_{j}, j=1,2, \ldots, n$. Thus the classical price trajectory $q_{j}(t)$, does not depend on dynamics of prices of shares for other traders $i \neq j$ (for example, the price of shares of ERIKSSON does not depend on the price of shares of NOKIA and vice versa). ${ }^{8}$

\footnotetext{
${ }^{7}$ The reader may be surprised that there appeared complex numbers $C$. However, the use of these numbers is just a mathematical trick that provides the simple mathematical description of dynamics of the financial pilot wave.

${ }^{8}$ Such a dynamics would be natural if these corporations operate on independent markets, e.g., ERIKSSON in Sweden and NOKIA in Finland. Prices of their shares would depend only on local market conditions, e.g., on capacities of markets or consuming activity.
} 
However, if, e.g.,

$$
\psi\left(q_{1}, \ldots, q_{n}\right)=c e^{i\left(q_{1} q_{2}+\ldots+q_{n-1} q_{n}\right)} e^{-\left(q_{1}^{2}+\ldots+q_{n}^{2}\right)},
$$

where $c \in C$ is some normalization constant, then financial behavior of agents at the financial market is nonlocal (see further considerations).

2. Reactions of the market do not depend on the amplitude of the financial pilot wave: waves $\psi, 2 \psi, 100000 \psi$ will produce the same reaction. Such a behavior at the market is quite natural (if the financial pilot wave is interpreted as an information wave, the wave of financial information). The amplitude of an information signal does not play so large role in the information exchange. The most important is the context of such a signal. The context is given by the shape of the signal, the form of the financial pilot wave function.

\section{The Dynamics of Prices Guided by the Fi- nancial Pilot Wave}

In fact, we need not develop a new mathematical formalism. We will just apply the standard pilot wave formalism to the financial market. The fundamental postulate of the pilot wave theory is that the pilot wave (field) $\psi\left(q_{1}, \ldots, q_{n}\right)$ induces a new (quantum) potential $U\left(q_{1}, \ldots, q_{n}\right)$ which perturbs the classical equations of motion. A modified Newton equation has the form:

$$
\dot{p}=f+g,
$$

where $f=-\frac{\partial V}{\partial q}$ and $g=-\frac{\partial U}{\partial q}$. We call the additional financial force $g$ a financial mental force. This force $g\left(q_{1}, \ldots, q_{n}\right)$ determines a kind of collective consciousness of the financial market. Of course, the $g$ depends on economic and other 'hard' conditions given by the financial potential $V\left(q_{1}, \ldots, q_{n}\right)$. However, this is not a direct dependence. In principle, a nonzero financial mental force can be induced by the financial pilot wave $\psi$ in the case of zero financial potential, $V \equiv 0$. So $V \equiv 0$ does not imply that $U \equiv 0$. Market psychology is not totally determined by economic factors. Financial (psychological) waves of information need not be generated by some changes in a real economic situation. They are mixtures of mental and economic 
waves. Even in the absence of economic waves, mental financial waves can have a large influence to the market.

By using the standard pilot wave formalism we obtain the following rule for computing the financial mental force. We represent the financial pilot wave $\psi(q)$ in the form:

$$
\psi(q)=R(q) e^{i S(q)}
$$

where $R(q)=|\psi(q)|$ is the amplitude of $\psi(q)$, (the absolute value of the complex number $c=\psi(q)$ ) and $S(q)$ is the phase of $\psi(q)$ (the argument of the complex number $c=\psi(q))$. Then the financial mental potential is computed as

$$
U\left(q_{1}, \ldots, q_{n}\right)=-\frac{1}{R} \sum_{i=1}^{n} \frac{\partial^{2} R}{\partial q_{i}^{2}}
$$

and the financial mental force as

$$
g_{j}\left(q_{1}, \ldots, q_{n}\right)=\frac{-\partial U}{\partial q_{j}}\left(q_{1}, \ldots, q_{n}\right) .
$$

These formulas imply that strong financial effects are produced by financial waves having essential variations of amplitudes.

Example 1. (Financial waves with small variation have no effect). Let $R \equiv$ const. Then the financial (behavioral) force $g \equiv 0$. As $R \equiv$ const, it is impossible to change expectations of the whole financial market by varying the price $q_{j}$ of one fixed type of shares, $j$. The constant information field does not induce psychological financial effects at all. As we have already remarked the absolute value of this constant does not play any role. Waves of constant amplitude $R=1$, as well as $R=10^{100}$, produce no effect.

Let $R(q)=c q, c>0$. This is a linear function; variation is not so large. As the result $g \equiv 0$ here also. No financial behavioral effects.

Example 2. (Speculation) Let

$$
R(q)=c\left(q^{2}+d\right), \quad c, d>0 .
$$

Here

$$
U(q)=-\frac{2}{q^{2}+d}
$$

(it does not depend on the amplitude $c$ !) and

$$
g(q)=\frac{-4 q}{\left(q^{2}+d\right)^{2}} .
$$


The quadratic function varies essentially more strongly than the linear function, and, as a result, such a financial pilot wave induces a nontrivial financial force.

We analyze financial drives induced by such a force. We consider the situation: (the starting price) $q>0$ and $g<0$. The financial force $g$ stimulates the market (which works as a huge cognitive system) to decrease the price. For small prices,

$$
g(q) \approx-4 q / d^{2} .
$$

If the financial market increases the price $q$ for shares of this type, then the negative reaction of the financial force becomes stronger and stronger. The market is pressed (by the financial force) to stop increasing of the price $q$. However, for large prices,

$$
g(q) \approx-4 / q^{3} .
$$

If the market can approach this range of prices (despite the negative pressure of the financial force for relatively small $q$ ) then the market will feel decreasing of the negative pressure (we recall that we consider the financial market as a huge cognitive system). This model explains well speculative behavior at the financial market.

Let

$$
R(q)=c\left(q^{4}+b\right), \quad c, b>0 .
$$

Thus

$$
g(q)=\frac{b q-q^{5}}{\left(q^{4}+b\right)^{2}} .
$$

Here behavior of the market is more complicated. Set $d={ }^{4} \sqrt{b}$. If the price $q$ is changing from $q=0$ to $q=d$ then the market is motivated (by the financial force $g(q))$ to increase the price. The price $q=d$ is critical for his financial activity. By psychological reasons (of course, indirectly based on the whole information available at the market) the market "understands" that it would be dangerous to continue to increase the price. After approaching the price $q=d$, the market has the psychological stimuli to decrease the price.

Financial pilot waves $\psi(q)$ with $R(q)$ that are polynomials of higher order can induce very complex behavior. The interval $[0, \infty)$ is split into a collection of subintervals $0<d_{1}<d_{2}<\ldots<d_{n}<\infty$ such that at each price level $q=d_{j}$ the trader changes his attitude to increase or to decrease the price.

In fact, we have considered just a one-dimensional model. In the real case we have to consider multidimensional models of huge dimension. A financial 
pilot wave $\psi\left(q_{1}, \ldots, q_{n}\right)$ on such a price space $Q$ induces splitting of $Q$ into a large number of domains $Q=O_{1} \cup \ldots \cup O_{N}$.

The only problem which we have still to solve is the description of the time-dynamics of the financial pilot wave, $\psi(t, q)$. We follow the standard pilot wave theory. Here $\psi(t, q)$ is found as the solution of Schrödinger's equation. The Schrödinger equation for the energy $H(q, p)=\frac{1}{2} \sum_{j=1}^{n} \frac{p_{j}^{2}}{m_{j}}+$ $V\left(q_{1}, \ldots, q_{n}\right)$ has the form:

$$
\begin{gathered}
i h \frac{\partial \psi}{\partial t}\left(t, q_{1}, \ldots, q_{n}\right)= \\
-\sum_{j=1}^{n} \frac{h^{2}}{2 m_{j}} \frac{\partial^{2} \psi\left(t, q_{1}, \ldots, q_{n}\right)}{\partial q_{j}^{2}}+V\left(q_{1}, \ldots, q_{n}\right) \psi\left(t, q_{1}, \ldots, q_{n}\right),
\end{gathered}
$$

with the initial condition $\psi\left(0, q_{1}, \ldots, q_{n}\right)=\psi\left(q_{1}, \ldots, q_{n}\right)$. Thus if we know $\psi(0, q)$ then by using Schrödinger's equation we can find the pilot wave at any instant of time $t, \psi(t, q)$. Then we compute the corresponding mental potential $U(t, q)$ and mental force $g(t, q)$ and solve Newton's equation.

We shall use the same equation to find the evolution of the financial pilot wave. We have only to make one remark, namely, on the role of the constant $h$ in Schrödinger's equation, cf. E. Haven [8], [9], 11]. In quantum mechanics (which deals with microscopic objects) $h$ is the Planck constant. This constant is assumed to play the fundamental role in all quantum considerations. However, originally $h$ appeared as just a scaling numerical parameter for processes of energy exchange. Therefore in our financial model we can consider $h$ as a price scaling parameter, namely, the unit in which we would like to measure price change. We do not present any special value for $h$. There are numerous investigations into price scaling. It may be that there can be recommended some special value for $h$ related to the modern financial market, a fundamental financial constant. However, it seems that $h=h(t)$ evolves depending on economic development.

We suppose that the financial pilot wave evolves via the financial Schrödinger equation (an analogue of Schrödinger's equation) on the price space. In the general case this equation has the form:

$$
i h \frac{\partial \psi}{\partial t}(t, q)=\widehat{H} \psi(t, q), \psi(0, q)=\psi(q)
$$

where $\widehat{H}$ is self-adjoint operator corresponding to the financial energy given 
by a function $H(q, p)$ on the financial phase space. Here we proceed in the same way as in ordinary quantum theory for elementary particles.

As the mathematical basis of the model we use the space $L_{2}(Q)$ of square integrable functions $\psi: Q \rightarrow \mathbf{C}$, where $Q$ is the configuration price space, $Q=R^{n}$, or some domain $Q \subset R^{n}$ (for example, $Q=R_{+}^{n}$ ) :

$$
\|\psi\|^{2}=\int_{Q}|\psi(x)|^{2} d x<\infty .
$$

Here $d x$ is the Lebesque measure, a uniform probability distribution, on the configuration price space. Of course, the uniform distribution $d x$ is not the unique choice of the normalization measure on the configuration price space. By choosing $d x$ we assume that in the absence of the pilot wave influence, e.g., for $\psi(x) \equiv$ const, all prices 'have equal rights'. In general, this is not true. If there is no financial (psychological) waves the financial market still strongly depends on 'hard' economic conditions. In general, the choice of the normalization measure $M$ must be justified by a real relation between prices. So in general the financial pilot wave $\psi$ belongs to the space $L_{2}(Q, d M)$ of square integrable functions with respect to some measure $M$ on the configuration price space: $\|\psi\|^{2}=\int_{Q}|\psi(x)|^{2} d M(x)<\infty$. In particular, $M$ can be a Gaussian measure: $d M(x)=\frac{1}{(2 \pi \operatorname{det} B)^{n / 2}} e^{\frac{-\left(B^{-1}(x-\alpha), x-\alpha\right)}{2}} d x$, where $B=\left(b_{i j}\right)_{i, j=1}^{n}$ is the covariance matrix and $\alpha=\left(\alpha_{1}, \ldots, \alpha_{n}\right)$ is the average vector. The measure $M$ describes classical random fluctuations in the financial market that are not related to 'quantum' effects. The latter effects are described in our model by the financial pilot wave. If the influence of this wave is very small we can use classical probabilistic models; in particular, based on the Gaussian distribution. The Gaussian model for price fluctuations was the first financial probabilistic model - Bachelier's model. In fact, Bachelier described price dynamics by Brownian motion. Therefore it would be even more natural to consider the Gaussian distribution of price changes. So it is useful to the study momentum representation for the pilot wave theory which was recently developed by B. Hiley [18]. Instead of financial waves on the configuration financial space $Q$ we can consider financial waves $\psi: P \rightarrow \mathbf{C}$ on the momentum space. We recall that the momentum $p=m v$, where $v$, the velocity, describes price changes. Therefore by following Bachelier we have to consider the Gaussian representation, $\psi \in L_{2}(P, d M)$, where $P$ is the price change space.

However, further investigations demonstrated that it seems that the Gaus- 
sian model for price changes is not the best one for describing price fluctuations. One of alternative models is based on the Levy process. Therefore it can be useful to investigate the 'quantum' financial model that is based on the Cauchy (Lorentzian) measure: $d M(p)=\frac{\gamma}{\pi} \frac{d p}{\gamma^{2}+p^{2}}$ on the momentum financial space. The Cauchy measure has some advantages. By using this measure we exclude from consideration financial waves which increase at infinity as a linear function. So $\psi(p) \approx p^{k}, k \geq 1, p \rightarrow \infty$, are excluded from this model. It looks very natural since prices (at least in the real financial market) can not change arbitrarily quickly.

We now turn back to the general scheme, concentrating on the configuration representation, $\psi: Q \rightarrow \mathbf{C} ; \psi \in L_{2}(Q) \equiv L_{2}(Q, d x)$. This is the general quantum-like statistical formalism on the price space.

As in ordinary quantum mechanics, we consider a representation of financial quantities, observables, by symmetric operators in $L_{2}(Q)$. By using Schrödinger's representation we define price and price change operators by setting: $\hat{q}_{j} \psi(q)=q_{j} \psi(q)$, the operator of multiplication by the $q_{j}$-price; $\hat{p}_{j}=\frac{h}{i} \frac{\partial}{\partial q_{j}}$, the operator of differentiation with respect to the $q_{j^{-}}$ price, normalized by the scaling constant $h$ (and $-i=\frac{1}{i}$ which provides the symmetry of $\hat{p}_{j}$ ). Operators of price and price change satisfy the canonical commutation relations: $[\hat{q}, \hat{p}]=\hat{q} \hat{p}-\hat{p} \hat{q}=i h$. By using this operator representation of price and price changes we can represent every function $H(q, p)$ on the financial phase-space as an operator $H(\hat{q}, \hat{p})$ in $L_{2}(Q)$. In particular, the financial energy operator is represented by the operator: $\hat{H}=-\sum_{j=1}^{n} \frac{h^{2}}{2 m_{j}} \frac{\partial^{2}}{\partial q_{j}^{2}}+V\left(q_{1}, \ldots, q_{n}\right)$. Here $V(q)$ is the multiplication operator by the function $V(q)$.

In this general quantum-like formalism for the financial market we do not consider individual evolution of prices. The theory is purely statistical. We can only determine the average of a financial observable $A$ for some fixed state $\phi$ of the financial market: $\langle A\rangle_{\phi}=\int_{Q} A(\phi)(x) \bar{\phi}(x) d x$. The use of the Bohmian model gives the additional possibility of determining individual trajectories.

\section{References}

[1] L. Bachelier, Theorie de la speculation, Ann. Sc. l'Ecole Normale Superiere 111-17, 21-86 (1890). 
[2] R. N. Mantegna and H. E. Stanley, it Introduction to econophysics (Cambridge, Cambridge Univ. Press, 2000).

[3] J. Soros, The alchemy of finance. Reading of mind of the market, J. Wiley and Sons, Inc.: New-York, 1987.

[4] L. Accardi, Urne e Camaleoni: Dialogo sulla realta, le leggi del caso e la teoria quantistica (Il Saggiatore, Rome, 1997)

[5] A. Yu. Khrennikov, Information dynamics in cognitive, psychological and anomalous phenomena (Kluwer, Dordreht, 2004).

[6] O. Choustova, Pilot wave quantum model for the stock market, http://www.arxiv.org/abs/quant-ph/0109122.

[7] E. Haven, A discussion on embedding the Black-Scholes option pricing model in a quantum physics setting, Physica A 304 (2002), 507-524.

[8] E. Haven, A Black-Sholes Schrödinger option price: 'bit'versus 'qubit', Physica A 324 (2003), 201-206.

[9] E. Haven, An ' $h$-Brownian motion' of stochastic option pices, Physica A 344 (2003), 151-155.

[10] W. Segal, I. E. Segal, Proc. Nat. Acad. Sc. USA 95 (1998), 4072-4075.

[11] E. Haven, Bohmian mechanics in a macroscopic quantum system. Foundations of Probability and Physics-3, ed. A. Yu. Khrennikov (Melville, New York: AIP Conference Proceedings, 2006).

[12] D. Bohm and B. Hiley, The undivided universe: an ontological interpretation of quantum mechanics, Routledge and Kegan Paul, London, 1993.

[13] B. Hiley, P. Pylkkänen, Active information and cognitive science - A reply to Kieseppä, n Brain, mind and physics. Pylkkänen, P., Pylkkö, P., Hautamäki, A., eds., IOS Press, Amsterdam, 1997.

[14] D. Bohm, Quantum theory, Prentice-Hall, Englewood Cliffs, NewJersey, 1951. 
[15] P. Holland, The quantum theory of motion, Cambridge Univ. Press, Cambridge, 1993.

[16] A. Yu. Khrennikov, Classical and quantum mechanics on information spaces with applications to cognitive, psychological, social and anomalous phenomena, Found. of Physics 29 (1999), 1065-1098.

[17] Khrennikov, A. Yu. Classical and quantum mechanics on p-adic trees of ideas. BioSystems 2000, 56, 95-120.

[18] B. Hiley, From the Heisenberg picture to Bohm: a new perspective on active information and its relation to Shannon information, in Quantum Theory: Reconsideration of Foundations, A. Yu. Khrennikov, ed., ser. Math. Modelling 10, Växjö University Press, Växjö, 2001. 